\title{
Reexamination of local spin polarization beyond global equilibrium in relativistic heavy ion collisions
}

\author{
Cong $\mathrm{Yi}^{*}$ and Shi Pu $\odot^{\dagger}$ \\ Department of Modern Physics, University of Science and Technology of China, Hefei, Anhui 230026, China \\ Di-Lun Yang $\mathbb{1}^{*}$ \\ Institute of Physics, Academia Sinica, Taipei 11529, Taiwan
}

(Received 9 June 2021; accepted 15 November 2021; published 6 December 2021)

\begin{abstract}
We study local spin polarization in the relativistic hydrodynamic model. Generalizing the Wigner functions previously obtained from chiral kinetic theory by Y. Hidaka et al. [Phys. Rev. D 97, 016004 (2018)] to the massive case, we present the possible contributions up to the order of $\hbar$ from thermal vorticity, shear viscous tensor, other terms associated with the temperature and chemical-potential gradients, and electromagnetic fields to the local spin polarization. We then implement the $(3+1)$-dimensional viscous hydrodynamic model to study the spin polarizations from these sources with a small chemical potential and ignorance of electromagnetic fields by adopting an equation of state different from those in other recent studies. Although the shear correction alone upon the local polarization results in a sign and azimuthal-angle dependence more consistent with experimental observations, as also discovered in other recent studies, it is mostly suppressed by the contributions from thermal vorticity and other terms that yield an opposite trend. It is found that the total local spin polarization can be very sensitive to the equation of states, the ratio of shear viscosity to entropy density, and the freeze-out temperature.
\end{abstract}

DOI: 10.1103/PhysRevC.104.064901

\section{INTRODUCTION}

In noncentral heavy ion collisions, large orbital angular momenta can be generated and transferred to the quark gluon plasma (QGP) in the form of vorticity fields. Proposed by Liang and Wang [1,2] in early pioneer works, the global orbital angular momenta could trigger the spin polarization of interacting partons through spin-orbit coupling. The magnitude of vorticity can be accordingly extracted through the spin polarization of hadrons created from the experiments. Later, relativistic fermions with spin at thermal equilibrium were systematically studied in the statistical model and a more precise relation to the possible experimental observation was established [3-5]. On the other hand, the properties and dynamical evolution of vorticity are further analyzed via numerical simulations in AMPT and HIJING [6-8]. Assuming the global equilibrium condition and employing the modified Cooper-Frye formula for spin polarization [5,9], the spin polarization of $\Lambda$ hyperons in heavy ion collisions has been estimated based on UrQMD [10] and hydrodynamic models [11-15]. See also Refs. [16-19] for other related

\footnotetext{
*congyi@mail.ustc.edu.cn

$\dagger$ shipu@ustc.edu.cn

‡lyang@gate.sinica.edu.tw
}

Published by the American Physical Society under the terms of the Creative Commons Attribution 4.0 International license. Further distribution of this work must maintain attribution to the author(s) and the published article's title, journal citation, and DOI. Funded by $S C O A P^{3}$. studies. The theoretic predictions and follow-up studies $[8,10,20-23]$ remarkably agree with the later measurement of the global polarization for $\Lambda$ and $\bar{\Lambda}$ hyperons by the STAR Collaboration [24]. It is also shown that the average angular velocity or vorticity of QGP is as large as $\omega \sim 10^{22} \mathrm{~s}^{-1}$ [24], which reveals that the QGP could be the most vortical fluid so far.

Despite the agreement between theory and experiment on global polarization, the STAR Collaboration has also measured the azimuthal angle dependence of the spin polarization along the beam and the out-of-plane directions in $\mathrm{Au}+\mathrm{Au}$ collisions at $200 \mathrm{GeV}[25,26]$, known as the longitudinal and transverse local spin polarization, respectively. Most theoretical simulations, e.g., relativistic hydrodynamics $[27,28]$ and transport models [10,21,29], have failed to describe the measurements of local polarization, with a few exceptions, such as the numerical simulation from the kinetic theory of massless fermions in Ref. [30] and results from some phenomenological models in Refs. [15], [31], and [32]. In general, these theoretical estimations have found local polarization with an opposite sign versus the experimental result. Also, feed-down effects are found to be negligible for recoiling the tension $[33,34]$. This disagreement between theory and experiment for local spin polarization is dubbed the "sign" problem.

Since most of the previous simulations have relied on the assumption that spin polarization for $\Lambda$ hyperons at the freeze-out hypersurface is mainly induced by the thermal vorticity in light of the statistical model [5] and Wignerfunction approach [35] at global equilibrium, it is generally believed that nonequilibrium effects beyond the assumption of global equilibrium are essential to delineate the local spin 
polarization. Consequently, many theoretical efforts have focused on studying dynamical spin polarization. One microscopic theory for tracking the dynamical evolution of spin transport for relativistic fermions is the quantum kinetic theory (QKT). The QKT was developed for massless fermions [36-52] at the very beginning, then known as the chiral kinetic theory (CKT). To describe the spin dynamics, in particular, of strange $(s)$ quarks or $\Lambda$ hyperons, QKT has been extended to the case of massive fermions [53-65]. In Ref. [66], another microscopic model for spin polarization through particle collisions is proposed. As for macroscopic descriptions, one may need to add the spin effects to the relativistic hydrodynamics, i.e., relativistic spin hydrodynamics [67-77]. Spin hydrodynamic has been derived from many approaches, e.g., the entropy principle $[72,78,79]$, the Lagrangian effective theory $[75,76]$, kinetic approaches $[67,68,70,71,73,74]$, and general discussion from field theory [80]. Also, see recent reviews [81-85] and the references therein.

Nevertheless, simulating the spin polarization of relativistic fermions far from equilibrium is technically difficult and computationally expensive. It is instead natural to explore the spin polarization near local equilibrium. From the detailed balance of massless fermions with two-to-two scattering in chiral kinetic theory, it has been shown that the Wigner function pertinent to spin polarization contains several corrections besides the thermal vorticity at local equilibrium as derived by some of the authors of this paper [36] in 2017. Recently, the authors of Refs. [86] and [87] have found similar contributions for massive fermions at local equilibrium, which have a smooth connection to part of the massless result. In Ref. [88], the authors have simultaneously derived the shearinduced polarization through statistical models. Based on their findings with particularly the shear correction, the hydrodynamic simulations in Ref. [89] show qualitative agreement with experimental data obtained by computing the polarization of $s$ quarks in $\Lambda$ hyperons. Simultaneously, the authors of Ref. [90] have added the contribution from the shear viscous tensor to the local spin polarization near isothermal equilibrium, and the numerical results also agree with the experimental data qualitatively.

A natural question then arises: Is the numerical finding above with the shear corrections sensitive to the parameters chosen in the hydrodynamics simulations? If the answer is positive, it implies that the correct "sign" for local polarization may not be solely attributed to the shear correction at local equilibrium, and further nonequilibrium corrections depending on interaction should be considered. Therefore, we follow the early work [36] done by some of the authors of the present paper and list all possible local-equilibrium corrections to the local spin polarization up to order $\hbar$. We then implement $(3+1)$-dimensional viscous hydrodynamics to investigate the polarization induced by these effects. In order to examine the dependence of the numerical parameters in simulations, we use an equation of state $(\mathrm{EoS})$ different from the one adopted in Ref. [89] and discuss the dependence of the freeze-out temperature and the ratio of shear viscosity to entropy density.

The structure of the paper is as follows. In Sec. II, we review the main results of our early work [36] and present all possible corrections at local equilibrium to polarization up to order $\hbar$ explicitly. Next, we implement viscous hydrodynamical simulations with the AMPT initial condition to study the polarization and discuss the dependence of the freeze-out temperature and the ratio of shear viscosity to entropy density in Sec. III. We summarize in Sec. IV.

Throughout this paper, we use the Minkowski metric $g_{\mu \nu}=$ $\operatorname{diag}\{+,-,-,-\}$ and define the Levi-Civita tensor $\epsilon^{\mu \nu \alpha \beta}$ with the convention $\epsilon^{0123}=-\epsilon_{0123}=1$. We also introduce the notations $A_{(\rho} B_{\sigma)} \equiv\left(A_{\rho} B_{\sigma}+A_{\sigma} B_{\rho}\right) / 2$ and $A_{[\rho} B_{\sigma]} \equiv\left(A_{\rho} B_{\sigma}-\right.$ $\left.A_{\sigma} B_{\rho}\right) / 2$.

\section{THEORETICAL ANALYSIS FROM QUANTUM KINETIC THEORIES}

We are interested in the polarization (pseudo-) vector characterized by the axial-charge current density in phase space,

$$
\mathcal{J}_{5}^{\mu}(p, X) \equiv 2 \int_{p \cdot n}\left[\mathcal{J}_{+}^{\mu}(p, X)-\mathcal{J}_{-}^{\mu}(p, X)\right],
$$

where $\int_{p \cdot n} \equiv \int d p \cdot n p \cdot n \theta(p \cdot n) /(2 \pi)$, with $\theta(p \cdot n)$ a unitstep function. ${ }^{1}$ Here $\mathcal{J}_{+}^{\mu}(p, X)$ and $\mathcal{J}_{-}^{\mu}(p, X)$ denote the Wigner functions for right- and left-handed fermions, respectively. Given $\mathcal{J}_{5}^{\mu}(p, X)$, we can calculate the spin polarization of $\Lambda$ hyperons via the modified Cooper-Frye formula [5],

$$
\mathcal{S}^{\mu}(\mathbf{p})=\frac{\int d \Sigma \cdot p \mathcal{J}_{5}^{\mu}(p, X)}{2 m_{\Lambda} \int d \Sigma \cdot \mathcal{N}(p, X)},
$$

where $\mathcal{N}^{\mu}(p, X) \equiv 2 \int_{p \cdot n}\left[\mathcal{J}_{+}^{\mu}(p, X)+\mathcal{J}_{-}^{\mu}(p, X)\right]$ is the number density in phase space, $m_{\Lambda}$ is the mass of $\Lambda$, and $\Sigma_{\mu}$ is the normal vector of the freeze-out surface.

As derived in the early work [36], the Wigner functions for right- or left-handed fermions near local equilibrium are given $\mathrm{by}^{2}$

$$
\begin{aligned}
\mathcal{J}_{\lambda}^{\mu}(p, X)= & 2 \pi \operatorname{sign}(u \cdot p)\left\{\delta\left(p^{2}\right) p^{\mu}+\lambda \frac{\hbar}{2} \delta\left(p^{2}\right)\left[u^{\mu}(p \cdot \omega)\right.\right. \\
& \left.-\omega^{\mu}(u \cdot p)-2 S_{(u)}^{\mu \nu} \tilde{E}_{\nu}\right] \partial_{u \cdot p} \\
& \left.+\lambda \frac{\hbar}{4} \epsilon^{\mu \nu \alpha \beta} F_{\alpha \beta} \partial_{v}^{p} \delta\left(p^{2}\right)\right\} f_{\lambda}^{(0)}
\end{aligned}
$$

where $\lambda= \pm$ for right- and left-handed fermions, $u^{\mu}$ is the fluid four velocity,

$$
\begin{aligned}
S_{(u)}^{\mu \nu}= & \epsilon^{\mu \nu \alpha \beta} p_{\alpha} u_{\beta} /(2 u \cdot p), \\
\tilde{E}_{v}= & E_{\nu}+T \partial_{\nu} \frac{\mu_{\lambda}}{T}+\frac{(u \cdot p)}{T} \partial_{\nu} T \\
& -p^{\sigma}\left[\partial_{<\sigma} u_{v>}+\frac{1}{3} \Delta_{\sigma \nu}(\partial \cdot u)+u_{\nu} D u_{\sigma}\right],
\end{aligned}
$$

\footnotetext{
${ }^{1}$ For computation of the spin polarization, we usually apply the on-shell Wigner functions. Here we thus integrate over the energy defined as $p \cdot n$, where $n^{\mu}$ may be chosen as the fluid four velocity at thermal equilibrium. Also, we further introduce a unit-step function to omit the contribution from antifermions.

${ }^{2}$ In general, there exist off-equilibrium corrections pertinent to collisions, which are, however, higher order in gradient expansion starting from $O(\partial)$ and $O\left(\partial^{2}\right)$ for even- and odd-parity terms, respectively. These corrections are neglected here.
} 
and

$$
f_{\lambda}^{(0)}=1 /\left(e^{\left(u \cdot p-\mu_{\lambda}\right) / T}+1\right)
$$

is the distribution function, with $T$ the local temperature and $\mu_{ \pm}$the chemical potentials for right- or left-handed fermions, respectively. When evaluating the current by integrating $\mathcal{J}_{\lambda}^{\mu}(p, X)$ over $p$, we may rewrite the last term in Eq. (3) as

$$
\begin{aligned}
& 2 \pi \operatorname{sign}(u \cdot p) \lambda \frac{\hbar}{4} \epsilon^{\mu \nu \alpha \beta} F_{\alpha \beta} \partial_{\nu}^{p} \delta\left(p^{2}\right) f_{\lambda}^{(0)} \\
& \approx-2 \pi \operatorname{sign}(u \cdot p) \lambda \frac{\hbar \delta\left(p^{2}\right)}{4} \epsilon^{\mu \nu \alpha \beta} F_{\alpha \beta} \partial_{p \nu} f_{\lambda}^{(0)}
\end{aligned}
$$

by dropping the surface term. The rewritten form allows us to properly introduce the axial-charge current density in phase space through Eq. (1). In general, $f_{\lambda}$ should incorporate nonequilibrium corrections depending on interaction to satisfy the kinetic equation near local equilibrium, while the corrections are expected to be higher order in the gradient expansion and are omitted here for simplicity $[36,91]$. Here, electromagnetic fields are defined in the fluid rest frame,

$$
E_{\mu} \equiv u^{\nu} F_{\mu \nu}, \quad B^{\mu} \equiv \frac{1}{2} \epsilon^{\mu \nu \alpha \beta} u_{\nu} F_{\alpha \beta} .
$$

We also decompose the derivative of $u_{v}$ as

$$
\partial_{\mu} u_{v}=\partial_{\langle\mu} u_{\nu\rangle}+u_{\mu} D u_{\nu}+\frac{1}{3} \Delta_{\mu \nu}(\partial \cdot u)+\omega_{\mu \nu},
$$

where

$$
\Delta^{\mu \nu}=g^{\mu \nu}-u^{\mu} u^{\nu}
$$

is the projector,

$$
D \equiv u \cdot \partial,
$$

$A^{\langle\mu \nu\rangle}$ is the traceless part of an arbitrary tensor, $A^{\mu \nu}$,

$$
A^{\langle\mu \nu\rangle} \equiv \frac{1}{2}\left[\Delta^{\mu \alpha} \Delta^{\nu \beta}+\Delta^{\nu \beta} \Delta^{\mu \alpha}\right] A_{\alpha \beta}-\frac{1}{3} \Delta^{\mu \nu} \Delta^{\alpha \beta} A_{\alpha \beta},
$$

and $\omega^{\mu v}$ is the vorticity tensor,

$$
\omega_{\alpha \beta}=\epsilon_{\alpha \beta \mu \nu} u^{\mu} \omega^{\nu}+\frac{1}{2}\left(u_{\alpha} D u_{\beta}-u_{\beta} D u_{\alpha}\right),
$$

with vorticity defined as

$$
\omega^{\mu}=\frac{1}{2} \epsilon^{\mu \nu \alpha \beta} u_{\nu} \partial_{\alpha} u_{\beta} .
$$

Using the relation,

$$
\begin{aligned}
& u^{\mu}(p \cdot \omega)-\omega^{\mu}(u \cdot p) \\
& \quad=-\frac{1}{2} \epsilon^{\mu \nu \alpha \beta} p_{\nu} \partial_{\alpha} u_{\beta}+\frac{1}{2} \epsilon^{\mu \nu \alpha \beta} p_{\nu} u_{\alpha} D u_{\beta},
\end{aligned}
$$

we find

$$
\begin{aligned}
u^{\mu}(p \cdot \omega)-\omega^{\mu}(u \cdot p)-2 S_{(u)}^{\mu \nu} \tilde{E}_{v} \\
=-\frac{1}{2} \epsilon^{\mu \nu \alpha \beta} p_{\nu} T \partial_{\alpha} \frac{u_{\beta}}{T}+\frac{1}{2} \epsilon^{\mu \nu \alpha \beta} p_{\nu} u_{\alpha} D u_{\beta} \\
\quad-\frac{1}{2} \epsilon^{\mu \nu \alpha \beta} p_{\alpha} u_{\beta} \frac{1}{T} \partial_{\nu} T \\
\quad-\frac{1}{(u \cdot p)} \epsilon^{\mu \nu \alpha \beta} p_{\alpha} u_{\beta} E_{\nu}-\frac{T}{(u \cdot p)} \epsilon^{\mu \nu \alpha \beta} p_{\alpha} u_{\beta} \partial_{\nu} \frac{\mu_{\lambda}}{T} \\
\quad+\frac{1}{(u \cdot p)} \epsilon^{\mu \nu \alpha \beta} p_{\alpha} u_{\beta} p^{\sigma} \partial_{\langle\sigma} u_{\nu\rangle} .
\end{aligned}
$$

For convenience, we decompose $\mathcal{J}_{5}^{\mu}$ as

$$
\mathcal{J}_{5}^{\mu}=\mathcal{J}_{\text {thermal }}^{\mu}+\mathcal{J}_{\text {shear }}^{\mu}+\mathcal{J}_{\text {accT }}^{\mu}+\mathcal{J}_{\text {chemical }}^{\mu}+\mathcal{J}_{\mathrm{EB}}^{\mu},
$$

where

$$
\begin{aligned}
\mathcal{J}_{\text {thermal }}^{\mu} & =a \frac{1}{2} \epsilon^{\mu \nu \alpha \beta} p_{\nu} \partial_{\alpha} \frac{u_{\beta}}{T}, \\
\mathcal{J}_{\text {shear }}^{\mu} & =-a \frac{1}{(u \cdot p) T} \epsilon^{\mu \nu \alpha \beta} p_{\alpha} u_{\beta} p^{\sigma} \partial_{\langle\sigma} u_{v\rangle}, \\
\mathcal{J}_{\text {accT }}^{\mu} & =-a \frac{1}{2 T} \epsilon^{\mu \nu \alpha \beta} p_{\nu} u_{\alpha}\left(D u_{\beta}-\frac{1}{T} \partial_{\beta} T\right), \\
\mathcal{J}_{\text {chemical }}^{\mu} & =a \frac{1}{(u \cdot p)} \epsilon^{\mu \nu \alpha \beta} p_{\alpha} u_{\beta} \partial_{\nu} \frac{\mu}{T}, \\
\mathcal{J}_{\mathrm{EB}}^{\mu} & =a \frac{1}{(u \cdot p) T} \epsilon^{\mu \nu \alpha \beta} p_{\alpha} u_{\beta} E_{\nu}+a \frac{B^{\mu}}{T},
\end{aligned}
$$

with

$$
a=4 \pi \hbar \operatorname{sign}(u \cdot p) \delta\left(p^{2}\right) f_{V}^{(0)}\left(1-f_{V}^{(0)}\right) .
$$

Note that

$$
f_{V}^{(0)}=\frac{1}{2}\left(f_{+}^{(0)}+f_{-}^{(0)}\right),
$$

and we have set the same chemical potential for both left and right fermions, $\mu=\mu_{+}=\mu_{-}$, for simplicity. The subscripts "thermal," "shear," "accT," "chemical," and "EB" stand for the terms related to the thermal vorticity, shear viscous tensor, fluid acceleration minus the gradient of temperature $D u_{\mu}-\left(\Delta_{\mu \nu} \partial^{\nu} T\right) / T$, gradient of $\mu / T$, and electromagnetic fields, respectively. Except for $\mathcal{J}_{\text {thermal }}^{\mu}$ and part of $\mathcal{J}_{\mathrm{EB}}^{\mu}$ led by magnetic fields, all other terms in $\mathcal{J}_{5}^{\mu}$ come from the corrections beyond global equilibrium.

Let us take a close look at $\mathcal{J}_{\text {accT }}^{\mu}$, which is usually neglected for the following reason. One may utilize hydrodynamic equations of motion up to the order of $\hbar$ or $\partial^{2}$,

$$
\begin{aligned}
D T & =-\frac{\epsilon+P}{p} \Delta^{\mu \nu} \partial_{\nu} u_{\mu}+O\left(\hbar, \partial^{2}\right), \\
D u_{\mu} & =\frac{\Delta_{\mu \nu} \partial^{v} P}{\epsilon+P}+O\left(\hbar, \partial^{2}\right), \\
D \bar{\mu}_{\mathrm{R} / \mathrm{L}} & =O\left(\hbar, \partial^{2}\right),
\end{aligned}
$$

to replace the temporal derivatives $D$ in thermodynamic parameters, where $\epsilon$ and $P$ correspond to the energy density and pressure, respectively. Here the $\hbar$ corrections in Eq. (20) are irrelevant since they only contribute to higher-order terms at $O\left(\hbar^{2}\right)$ in Wigner functions except for the off-equilibrium fluctuations led by collisions.

In the ideal limit, one of the hydrodynamic equations of motion becomes $D u_{\mu}=\left(\Delta_{\mu \nu} \partial^{\nu} T\right) / T$. Therefore, $\mathcal{J}_{\text {accT }}^{\mu}$ vanishes in the ideal hydrodynamics. In realistic hydrodynamic simulations, dissipative corrections could further modify the evolution of $D u_{\mu}$. In viscous hydrodynamics, we have

$$
\begin{aligned}
D u_{\alpha} & =\frac{1}{\epsilon+P} \Delta_{\mu \alpha} \partial^{\mu} P-\frac{1}{\epsilon+P} \Delta_{\mu \alpha} \partial_{\nu} \pi^{\mu \nu}+O\left(\hbar, \partial^{3}\right) \\
& \approx \frac{\Delta_{\mu \alpha}}{T}\left(\partial^{\mu} T-s^{-1} \partial_{\nu} \pi^{\mu \nu}\right)
\end{aligned}
$$


where $\pi^{\mu \nu}=2 \Delta^{\mu \alpha} \Delta^{\nu \beta} \eta\left(\partial_{(\alpha} u_{\beta)}-g_{\alpha \beta} \partial_{\rho} u^{\rho} / 3\right)+O\left(\partial^{2}\right)$ corresponds to the shear-stress tensor, with $\eta$ being the shear viscosity and $s$ denoting the entropy density. Here we take $\epsilon+P=T s$ and omit the correction from the bulk viscosity for simplicity. We can further rewrite $\mathcal{J}_{\text {accT }}^{\mu}$ as

$$
\begin{aligned}
\mathcal{J}_{\mathrm{accT}}^{\mu} & =a \frac{1}{2 T^{2} s} \epsilon^{\mu \nu \alpha \beta} p_{\nu} u_{\alpha} \partial^{\rho} \pi_{\beta \rho} \\
& \approx a \frac{\eta}{T^{2} s^{2}} \epsilon^{\mu \nu \alpha \beta} p_{\nu} u_{\alpha} \partial^{\rho}\left(s \hat{\pi}_{\beta \rho}\right),
\end{aligned}
$$

where $\hat{\pi}^{\mu \nu}=\pi^{\mu \nu} /(2 \eta)$ and we have assumed that $\eta / s$ is a constant to make the final approximation above. Conse- quently, we would like to emphasize here that the contributions from $\mathcal{J}_{\text {acc }}^{\mu}$ to the local spin polarization should depend on the EoS and parameter $\eta / s$. Note that in early work [92], the authors discussed the contribution from fluid acceleration $D u_{\beta}$ implicitly involved in $\mathcal{J}_{\text {thermal }}^{\mu}$ to local polarization. Additionally, the magnetic-field contribution in $\mathcal{J}_{\mathrm{EB}}^{\mu}$ has also been discussed in Ref. [9].

Although we discuss the polarization of massless fermions above, we can extend our analysis to massive fermions. In the case of massive fermions, we can generalize the on-shell condition $\delta\left(p^{2}\right)$ in Eq. (18) to $\delta\left(p^{2}-m_{i}^{2}\right)$, with $m_{i}$ being the mass of fermions. Then we obtain $\mathcal{S}^{\mu}$ in Eq. (2) from different sources,

$$
\begin{aligned}
\mathcal{S}_{\text {thermal }}^{\mu}(\mathbf{p}) & =\frac{\hbar}{8 m_{\Lambda} N} \int d \Sigma^{\sigma} p_{\sigma} f_{V}^{(0)}\left(1-f_{V}^{(0)}\right) \epsilon^{\mu \nu \alpha \beta} p_{\nu} \partial_{\alpha} \frac{u_{\beta}}{T} \\
\mathcal{S}_{\text {shear }}^{\mu}(\mathbf{p}) & =-\frac{\hbar}{4 m_{\Lambda} N} \int d \Sigma \cdot p f_{V}^{(0)}\left(1-f_{V}^{(0)}\right) \frac{\epsilon^{\mu \nu \alpha \beta} p_{\alpha} u_{\beta}}{(u \cdot p) T} \frac{1}{2} p^{\sigma}\left[\left(\partial_{\sigma} u_{\nu}+\partial_{\nu} u_{\sigma}\right)-u_{\sigma} D u_{\nu}\right], \\
\mathcal{S}_{\text {accT }}^{\mu}(\mathbf{p}) & =-\frac{\hbar}{8 m_{\Lambda} N} \int d \Sigma \cdot p f_{V}^{(0)}\left(1-f_{V}^{(0)}\right) \frac{1}{T} \epsilon^{\mu \nu \alpha \beta} p_{\nu} u_{\alpha}\left(D u_{\beta}-\frac{1}{T} \partial_{\beta} T\right), \\
\mathcal{S}_{\text {chemical }}^{\mu}(\mathbf{p}) & =\frac{\hbar}{4 m_{\Lambda} N} \int d \Sigma \cdot p f_{V}^{(0)}\left(1-f_{V}^{(0)}\right) \frac{1}{(u \cdot p)} \epsilon^{\mu \nu \alpha \beta} p_{\alpha} u_{\beta} \partial_{\nu} \frac{\mu}{T}, \\
\mathcal{S}_{\mathrm{EB}}^{\mu}(\mathbf{p}) & =\frac{\hbar}{4 m_{\Lambda} N} \int d \Sigma \cdot p f_{V}^{(0)}\left(1-f_{V}^{(0)}\right)\left(\frac{1}{(u \cdot p) T} \epsilon^{\mu \nu \alpha \beta} p_{\alpha} u_{\beta} E_{v}+\frac{B^{\mu}}{T}\right),
\end{aligned}
$$

where $N=\int d \Sigma^{\mu} p_{\mu} f_{V}^{(0)}$ and now $p^{0}=\sqrt{|\mathbf{p}|^{2}+m_{i}^{2}}$. In this work, we only evaluate the spin polarization of $\Lambda$ and neglect $\bar{\Lambda}$.

We would like to emphasize that at global equilibrium it is necessary to impose a vanishing shear viscous strength to satisfy the free-streaming kinetic theory, which is equivalent to the Killing condition. On the other hand, at local equilibrium, one can also decompose $\partial_{\mu} u_{v}$ into more terms as opposed to our decomposition in Eq. (8), while the total effect led by the fluid-velocity gradient at local equilibrium should remain unchanged.

\section{RESULTS AND DISCUSSION}

\section{A. Setup for simulations}

In this section, we implement $(3+1)$-dimensional viscous hydrodynamic CLVisc $[93,94]$ with AMPT initial conditions $[15,32,95]$ to generate the freeze-out hypersurface and the profile of the fluid velocity and temperature at that hypersurface. Unless noted otherwise, we choose the EoS "s95p-pce" [96] instead of the EoS [97] used in Refs. [28] and [89].

We introduce the polarization along the beam direction as $\mathcal{P}^{z}(p)$ and that along the out-of-plane direction as $\mathcal{P}^{y}(p)$; they are obtained by integrating over the medium rapidity range $[-1,+1]$,

$$
\mathcal{P}^{z}(p)=\int_{-1}^{+1} d Y \mathcal{S}^{z}(p)
$$

$$
\mathcal{P}^{y}(p)=\int_{-1}^{+1} d Y \mathcal{S}^{y}(p),
$$

where $\mathcal{S}^{\mu}$ is given by Eqs. (23). We again use the subscripts "thermal," "shear," "accT," "chemical," and "EB" for $\mathcal{P}^{\mu}(p)$ with $\mu=y, z$ to specify the contributions to the polarization from the thermal vorticity, shear viscous tensor, $D u_{\beta}-\frac{1}{T} \partial_{\beta} T$, gradient of $\mu / T$, and electromagnetic fields, respectively.

Since the electromagnetic fields decay rapidly [98-100], we can neglect the contributions from electromagnetic fields $\mathcal{S}_{\mathrm{EB}}^{\mu}$ to the polarization vector $\mathcal{P}_{\mathrm{EB}}^{\mu}$. Due to the limitation of the EoS $s 95 p$-pce, we cannot get sufficient information on the chemical potential and its gradient. Therefore, in the current study, we only consider the polarization induced by the thermal vorticity $\mathcal{S}_{\text {thermal }}^{i}$, shear viscous tensor $\mathcal{S}_{\text {shear }}^{i}$, and $\mathcal{S}_{\text {accT }}^{i}$ and evaluate

$$
\mathcal{P}_{\text {total }}^{\mu}=\mathcal{P}_{\text {thermal }}^{\mu}+\mathcal{P}_{\text {shear }}^{\mu}+\mathcal{P}_{\text {accT }}^{\mu} .
$$

The possible contribution from $\mathcal{S}_{\text {chemical }}^{\mu}$ is also briefly discussed later.

We consider two scenarios in this work. In the first scenario, since the $\Lambda$ hyperons are produced at the chemical freeze-out, we assume that one can still utilize the macroscopic variables from hydrodynamics to describe the thermodynamical states of $\Lambda$ hyperons, e.g., the temperature and its gradient, i.e., we assume that $\Lambda$ hyperons are almost at local equilibrium. We call this the $\Lambda$ equilibrium scenario for short.

In the second scenario, as proposed in Ref. [89], since the spin of the $s$ quarks dominates the total spin of the $\Lambda$ hyperons 

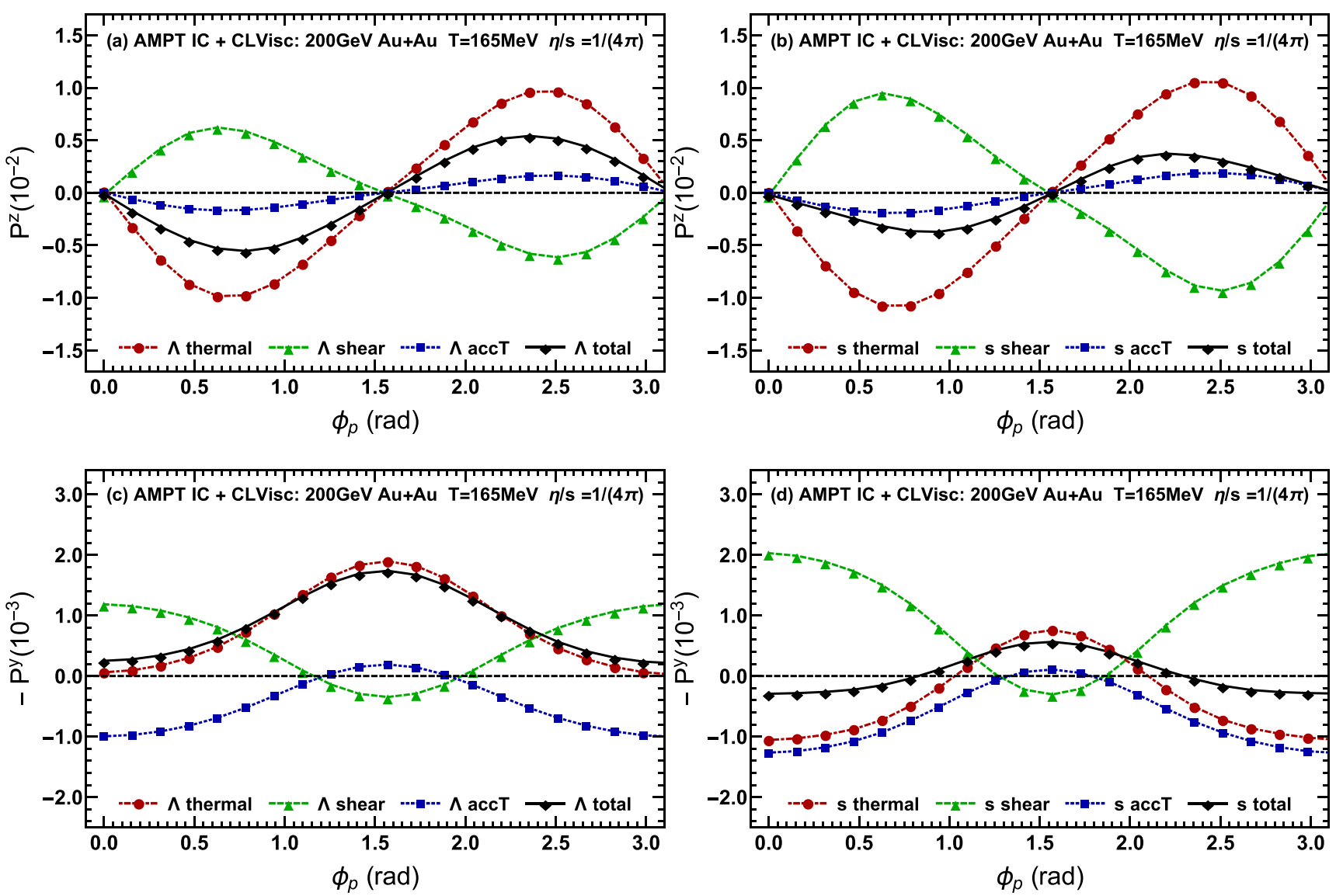

FIG. 1. The polarization $\mathcal{P}^{z}$ and $\mathcal{P}^{y}$ as a function of $\phi_{p}$ for the $\Lambda(\mathrm{a}, \mathrm{c})$ and $s(\mathrm{~b}, \mathrm{~d})$ equilibrium scenarios. We have chosen $\eta / s=1 /(4 \pi)$ and the freeze-out temperature is $165 \mathrm{MeV}$. Red, green, and blue curves represent the contributions from the thermal vorticity, shear-induced polarization, and acceleration terms, respectively. The black curve denotes the total polarization.

in the parton model, one can compute the polarization of $s$ quarks and assume that the spin polarization of the $s$ quark is smoothly passed to the polarization of $\Lambda$. We call this the $s$ equilibrium scenario. Since $s$ quarks are much lighter than $\Lambda$ hyperons, polarization induced by the shear viscous tensor will be greatly enhanced in the $s$ equilibrium scenario as opposed to the $\Lambda$ equilibrium scenario, where $\mathcal{S}_{\text {shear }}^{\mu}$ is suppressed by $(u \cdot p) \sim m_{\Lambda}$ in the denominator shown in Eq. (23).

Note that the polarization vectors in Eq. (24) are shown in the laboratory frame. In order to compare the results with the experimental data, we eventually transform them to the rest frame of the $\Lambda$ hyperon in the $\Lambda$ equilibrium scenario or of the $s$ quark in the $\Lambda$ hyperon in the $s$ equilibrium scenario. In principle, we also need to consider the evolution of $\Lambda$ hyperons before kinetic freeze-out, whereas we neglect the evolution of $\Lambda$ hyperons after the chemical freeze-out for simplicity.

We would like to emphasize that we always choose the factor $m$ in the denominator on the right-hand side of Eq. (2) as $m_{\Lambda}$ in both the $\Lambda$ and the $s$ equilibrium scenarios. The mass factor in Ref. [89] is instead chosen as $m_{s}$ in the $s$ equilibrium scenario, which will enhance the overall magnitude of polarization in the $s$ equilibrium scenario. The reason that we still choose overall factor $m$ in in the denominator on the right-hand side of Eq. (2) as $m_{\Lambda}$ in the $s$ equilibrium scenario is as follows. Although it is assumed that polarization of the $s$ quark dominates over other contributions in a $\Lambda$ hyperon in the $s$ equilibrium scenario, $\mathcal{S}^{\mu}(\mathbf{p})$ still corresponds to the polarization of the $\Lambda$ hyperon instead of the $s$ quark. Nevertheless, this choice only changes the magnitude of polarizations and does not change the qualitative results or main conclusion. For numerical simulations, we choose $m_{\Lambda}=1.116 \mathrm{GeV}$ for the mass of $\Lambda$ hyperons and $m_{s}=0.3 \mathrm{GeV}$ for the mass of constituent $s$ quarks.

\section{B. Numerical results and discussion}

Here we briefly summarize the results presented in each figure. In Fig. 1, we show the local spin polarization coming from different sources in the local rest frame. In Fig. 2, we compare the results in the particles' rest frames to those in Fig. 1. In Fig. 3, we present the results with a reduced freeze-out temperature. In Fig. 4, the results with a larger $\eta / s$ but the same freeze-out temperature as in Fig. 1 are presented. Finally, we show the spin polarization led by a small and constant chemical potential from $\mathcal{P}_{\text {chemical }}^{\mu}$ for a heuristic discussion. The detailed results and discussion are presented below.

In Fig. 1, we plot the local spin polarization in both the $\Lambda$ and the $s$ equilibrium scenarios with $\eta / s=1 /(4 \pi)$ and the 

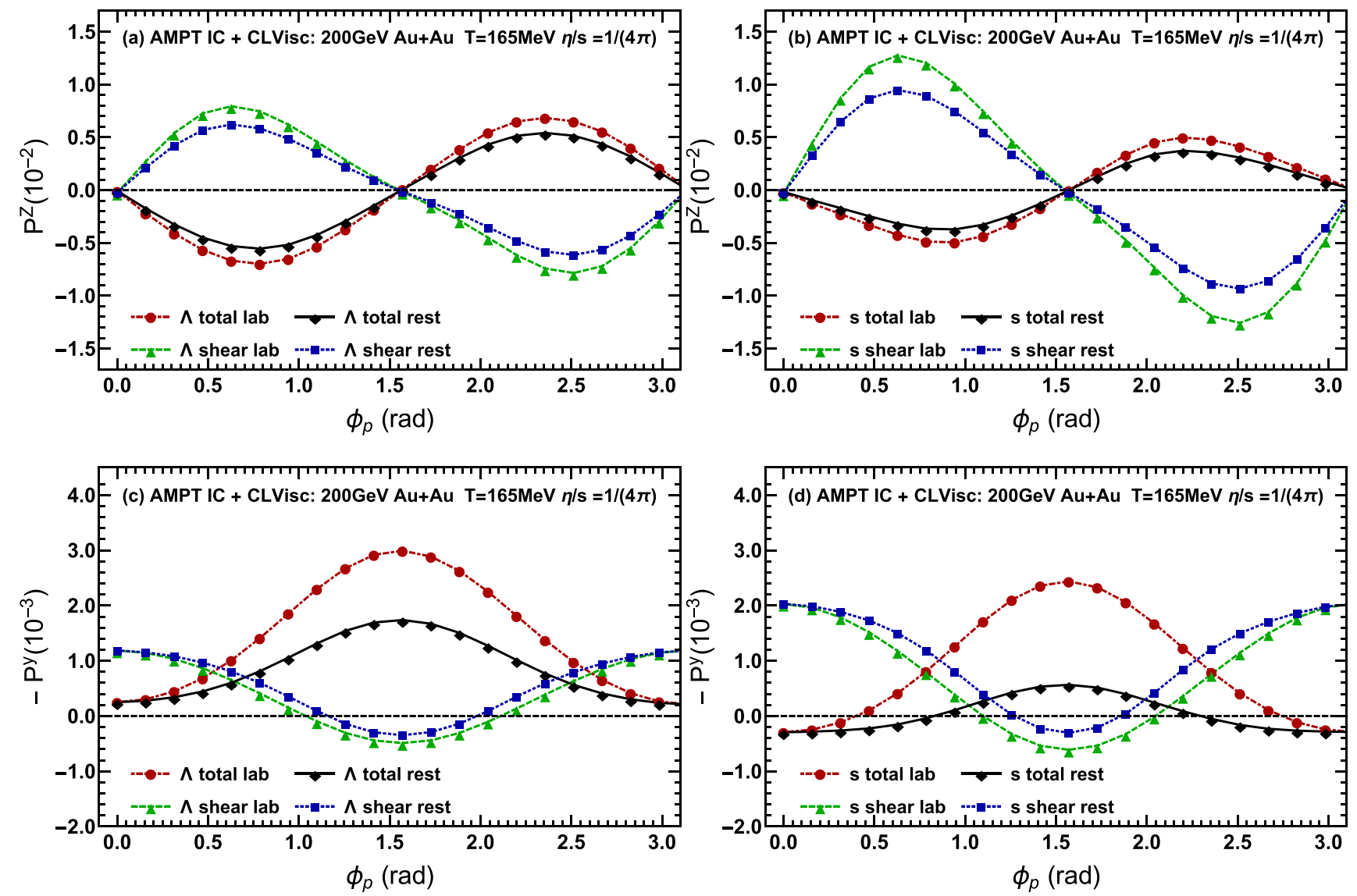

FIG. 2. The polarization $\mathcal{P}^{z}$ and $\mathcal{P}^{y}$ as a function of $\phi_{p}$ for $\Lambda$ hyperons and $s$ quarks in the laboratory frame and their own rest frames. We have chosen $\eta / s=1 /(4 \pi)$ and the freeze-out temperature is $165 \mathrm{MeV}$. Red and black curves represent the total polarization in the laboratory and the $\Lambda$ (or $s$ quark) rest frames, respectively. Green and blue curves denote the shear-induced polarization in the laboratory and the $\Lambda$ (or the $s$ quark) rest frames, respectively.

freeze-out temperature $T=165 \mathrm{MeV}$. We observe that $\mathcal{S}_{\text {shear }}^{\mu}$ always leads to the "same" sign contribution, qualitatively consistent with experimental data, to the local spin polarization both in the $z$ and $y$ directions and in the two scenarios. This observation is in accordance with Ref. [89]. Similarly to the thermal vorticity, $\mathcal{S}_{\text {accT }}^{\mu}$ induced by a nonvanishing $D u_{\mu}-\left(\Delta_{\mu \nu} \partial^{\nu} T\right) / T$ leads to the "opposite" sign contribution to the local polarization. Along the beam direction, the magnitude of polarization $\mathcal{P}_{\text {acc }}^{z}$ is much smaller than the magnitudes of $\mathcal{P}_{\text {thermal }}^{z}$ and $\mathcal{P}_{\text {shear }}^{z}$ in the two scenarios. Along the outof-plane direction, the $\mathcal{P}_{\text {acc }}^{y}$ is almost canceled by $\mathcal{P}_{\text {shear }}^{y}$ in the $\Lambda$ equilibrium scenario, while $\left|\mathcal{P}_{\text {acc }}^{y}\right|<\left|\mathcal{P}_{\text {shear }}^{y}\right|$ in the $s$ equilibrium scenario. As a consequence of the competition between $\mathcal{P}_{\text {shear }}^{\mu}$ and $\mathcal{P}_{\text {thermal }}^{\mu}+\mathcal{P}_{\text {accT }}^{\mu}$, the $\mathcal{P}_{\text {total }}^{z, y}$ in both the $\Lambda$ and the $s$ equilibrium scenarios disagree with the experimental data.

In contrast, the hydrodynamic simulations in Ref. [89] find that the $\mathcal{P}_{\text {total }}^{\mu}$ in the $s$ equilibrium scenarios agree with the experimental data qualitatively. As mentioned previously, we have chosen a different EoS than the one in Ref. [89].

One possible reason leading to the different results may derive from the following fact. In the EoS sp95-pce, the speed of sound connecting the QGP phase and PCE hadronic phase is not smooth and may lead to an unphysical vorticity structure near the freeze-out surface. We note that the authors in Ref. [28] have studied the EoS dependence on the global polarization.

We have also checked the results by using another EoS, lattice-wb2014 [101], and the total polarization has a similar "sign" as the experimental data qualitatively. But $\mathcal{P}_{\text {total }}^{z, y}$ as a function of $\phi_{p}$ are almost flat and $\left|\mathcal{P}_{\text {total }}^{z}\right|$ is quantitatively close to 0 due to the high suppression led by the factor $m_{\Lambda}$ in the denominator on the right-hand side of Eq. (2). Therefore, our results show that spin polarizations with local-equilibrium corrections from hydrodynamic simulations are sensitive to the choice of EoS.

In Fig. 2, we compare the polarization in the laboratory frame and in the particles' rest frames. It is found that the difference between the polarization in the two frames is slight for $\mathcal{P}_{\text {total }}^{z}$. Although the overall magnitudes and peaks of $\mathcal{P}_{\text {total }}^{y}$ are reduced in the particles' rest frames, in general, the choices of frames do not change the polarization qualitatively.

Next, we consider the dependence of the freeze-out temperature. We set the freeze-out temperature $T=157 \mathrm{MeV}$, keep $\eta / s=1 /(4 \pi)$, and show the results in Fig. 3. The magnitudes of $\mathcal{P}_{\text {acc }}^{\mu}$ increase significantly when the freeze-out 

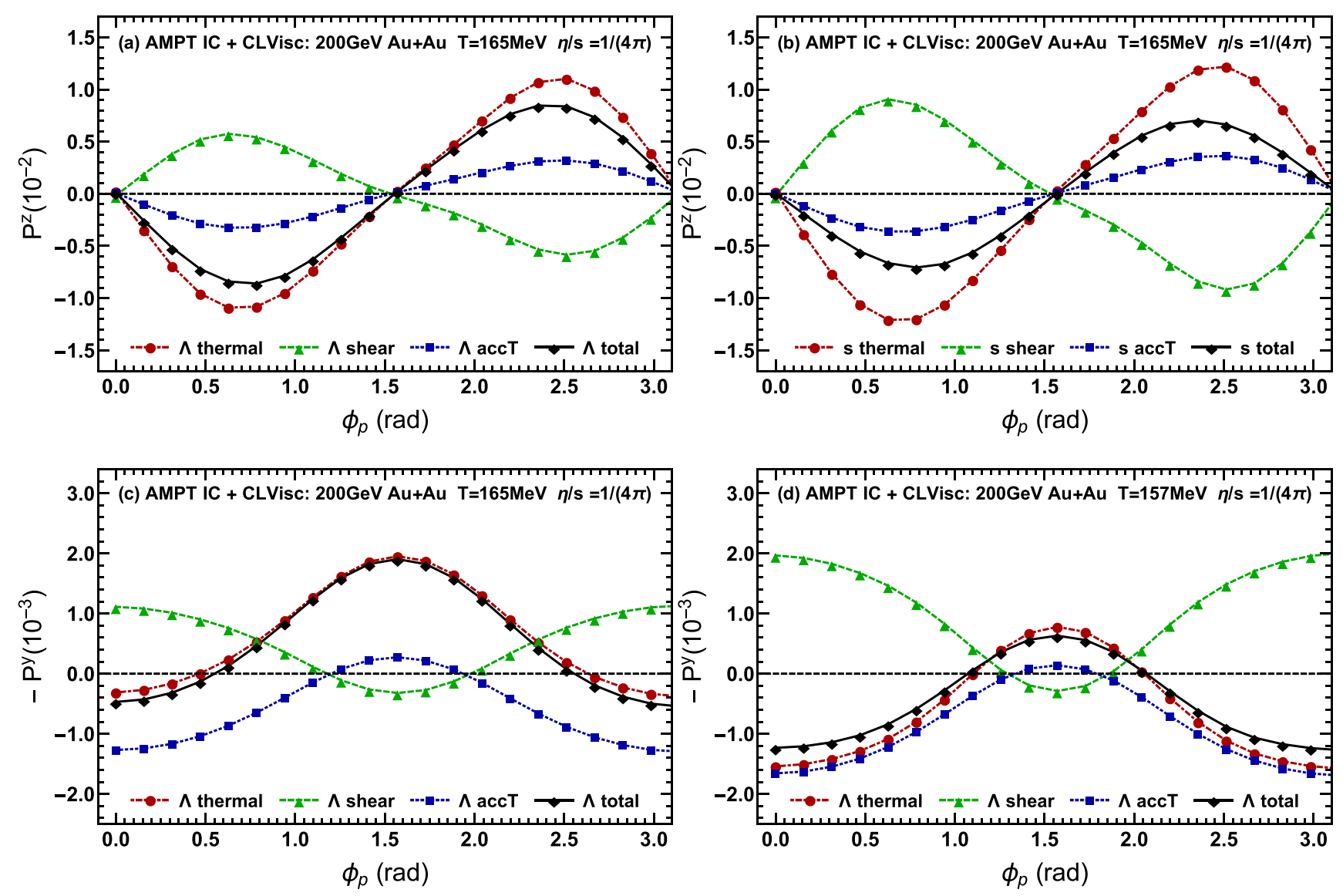

FIG. 3. The same setup and color assignments as in Fig. 1 except the freeze-out temperature has been changed to $157 \mathrm{MeV}$.

temperature decreases. ${ }^{3}$ Since now $\left|\mathcal{J}_{\text {accT }}^{\mu}\right| \sim O\left(T^{-2} p \partial^{2}\right)$ is a crude estimation for the magnitude of $\mathcal{J}_{\text {accT }}^{\mu}$ coming from the dissipative correction, it may be expected that $\left|\mathcal{P}_{\text {thermal }}^{\mu}\right|$ should increase with the reduced freeze-out temperature.

On the other hand, we observe that the shear-induced polarization $\mathcal{P}_{\text {shear }}^{\mu}$ is not sensitive to the freeze-out temperature, while the magnitude of the thermal vorticity-induced polarization $\mathcal{P}_{\text {thermal }}^{\mu}$ is slightly enhanced. The difference between $\mathcal{P}_{\text {total }}^{\mu}$ in the two scenarios and experimental data increases due to the increase in the magnitude of $\mathcal{P}_{\text {accT }}^{\mu}$. It turns out that $\mathcal{P}_{\text {total }}^{\mu}$ is also sensitive to the freeze-out temperature.

Third, we discuss the dependence of $\eta / s$. In Fig. 4, we set $\eta / s=2 /(4 \pi)$ and the freeze-out temperature $T=165 \mathrm{MeV}$. In comparison with the case where $\eta / s=1 /(4 \pi)$ in Fig. 1, we find that none of the contributions to $\mathcal{P}^{z}$ in the two scenarios have changed much. On the contrary, the magnitudes of both $\mathcal{P}_{\text {thermal }}^{y}$ and $\mathcal{P}_{\text {accT }}^{y}$ in the two scenarios increase when $\eta / s$ grows. The magnitudes of $\mathcal{P}_{\text {shear }}^{y}$ in the two scenarios become smaller than those in Fig. 1. Eventually, the $\mathcal{P}_{\text {total }}^{y}$ values in

\footnotetext{
${ }^{3}$ In fact, the magnitude of $\mathcal{P}_{\text {thermal }}^{\mu}$ also depends on the freeze-out temperature since $\mathcal{J}_{\text {thermal }}^{\mu}$ implicitly incorporates the term related to $D u^{\mu}$. But this term has been previously included in the hydrodynamic simulations for $\mathcal{P}_{\text {thermal }}^{\mu}$ at global equilibrium. It is noteworthy that the full Wigner function at local equilibrium, derived in Ref. [36], actually does not contain a term associated with $D u^{\mu}$.
}

the two scenarios are still different from the observations in experiments. It is found that the local polarization, at least for $\mathcal{P}_{\text {total }}^{y}$, also depends on $\eta / s$.

At last, we compute the possible contribution from the gradient of the chemical potential over the temperature led by $\mathcal{S}_{\text {chemical }}^{\mu}$ in Eq. (23). For simplicity, we assume that the chemical potential is constant near the freeze-out hypersurface and $\nabla \mu / T \simeq \mu \nabla(1 / T)$. In Fig. 5, we choose the quark chemical potential $\mu=10 \mathrm{MeV}$. Similarly to the shear viscous tensor, $\mathcal{S}_{\text {chemical }}^{\mu}$ leads to the "same" sign contribution, qualitatively consistent with the experimental data, to the local spin polarization both in the $z$ and $y$ directions and in the two scenarios. However, $\mathcal{P}_{\text {chemical }}^{\mu} \propto \mu$ is greatly suppressed by other contributions to $\mathcal{P}_{\text {total }}^{\mu}$. So far, they are almost negligible in the current study. However, $\mathcal{P}_{\text {chemical }}^{\mu}$ might be important in low-energy collisions. We leave this for future studies.

Before ending this section, we also comment on the choice of $m_{s}$. So far, there are no first principle calculations to demonstrate how large $m_{s}$ should be in these simulations. In general, one can set $m_{s}$ as the current quark mass or constituent quark mass different from $0.3 \mathrm{GeV}$. As discussed in this section, we have shown that $\mathcal{P}_{\text {shear }}^{\mu}$ competes with $\mathcal{P}_{\text {thermal }}^{\mu}$ and $\mathcal{P}_{\text {accT }}^{\mu}$. From Eq. (23), we find that the denominator $(u \cdot p) \sim p^{0} \sim m_{i}$ in $\mathcal{S}_{\text {shear }}^{\mu}$ is sensitive to the mass $m_{i}$. Since $m_{i}=m_{s}$ in the $s$ equilibrium scenario is much smaller than $m_{i}=m_{\Lambda}$ in the $\Lambda$ equilibrium scenario, $\mathcal{P}_{\text {shear }}^{\mu}$ is therefore significantly enhanced in the $s$ equilibrium scenario. Moreover, if we increase 

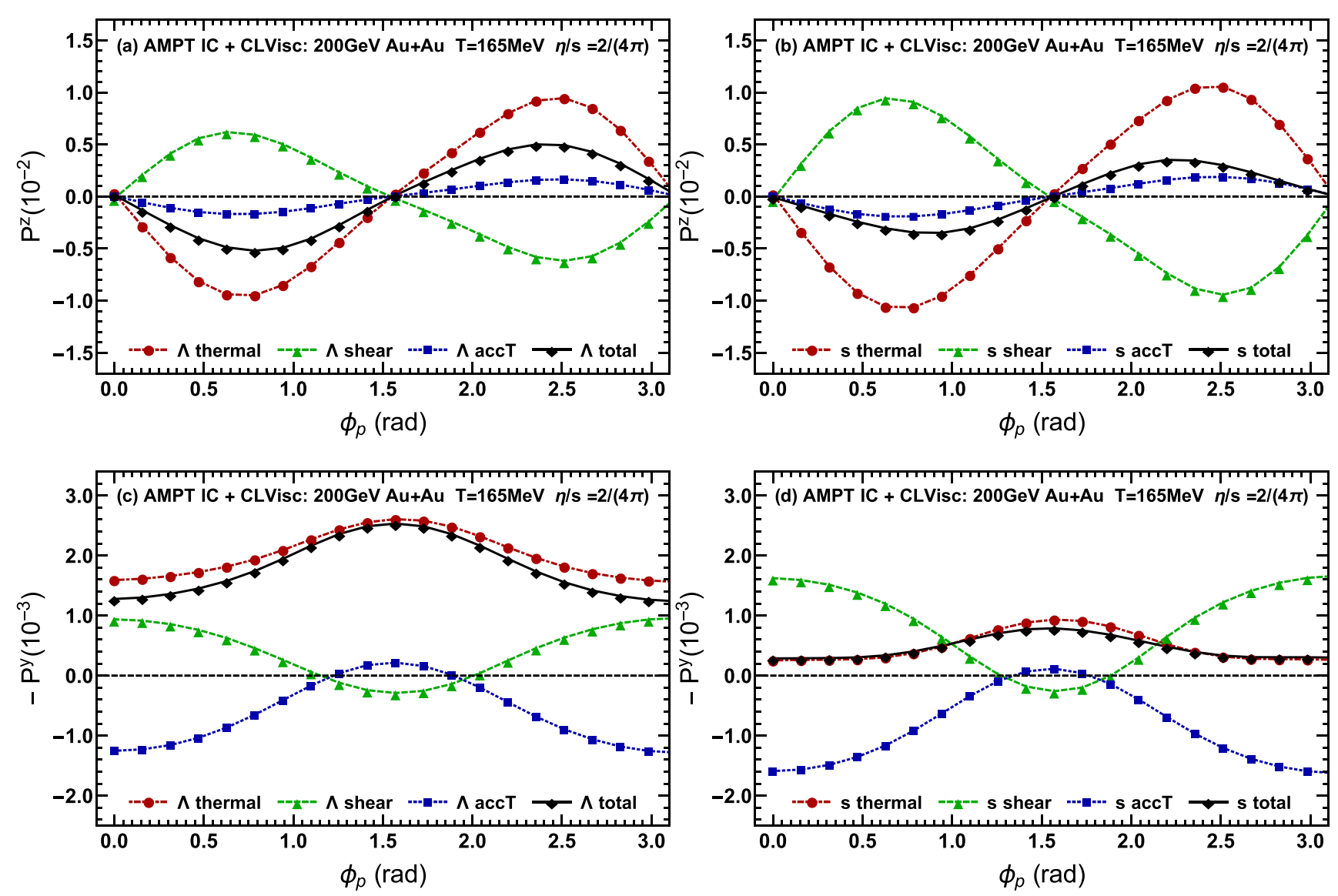

FIG. 4. The same setup and color assignments as in Fig. 1 except the shear viscosity-to-entropy density ratio has been changed to $\eta / s=$ $2 /(4 \pi)$.

$m_{s}, \mathcal{P}_{\text {shear }}^{\mu}$ obviously decreases. A more rigorous study of the spin fragmentation from $s$ quarks to $\Lambda$ hyperons in the hadronization process is required to resolve this uncertainty in the future.

As a concluding remark, we have shown that $\mathcal{P}_{\text {total }}^{\mu}$ is generally sensitive to the EoS, freeze-out temperature $T$, and ratio $\eta / s$. We find that both $\mathcal{S}_{\text {shear }}^{\mu}$ and $\mathcal{S}_{\text {accT }}^{\mu}$ play important roles in local spin polarization. The behavior of $\mathcal{P}_{\text {total }}^{\mu}$ greatly depends on the predominant term in the competition between $\mathcal{P}_{\text {shear }}^{\mu}$ and $\mathcal{P}_{\text {thermal }}^{\mu}+\mathcal{P}_{\text {accT }}^{\mu}$. Although $\mathcal{S}_{\text {shear }}^{\mu}$ may lead to the "same" sign of the polarization, we still cannot get a similar azimuthal angle dependence of the local spin polarization as the experimental data with the EoS we have chosen.
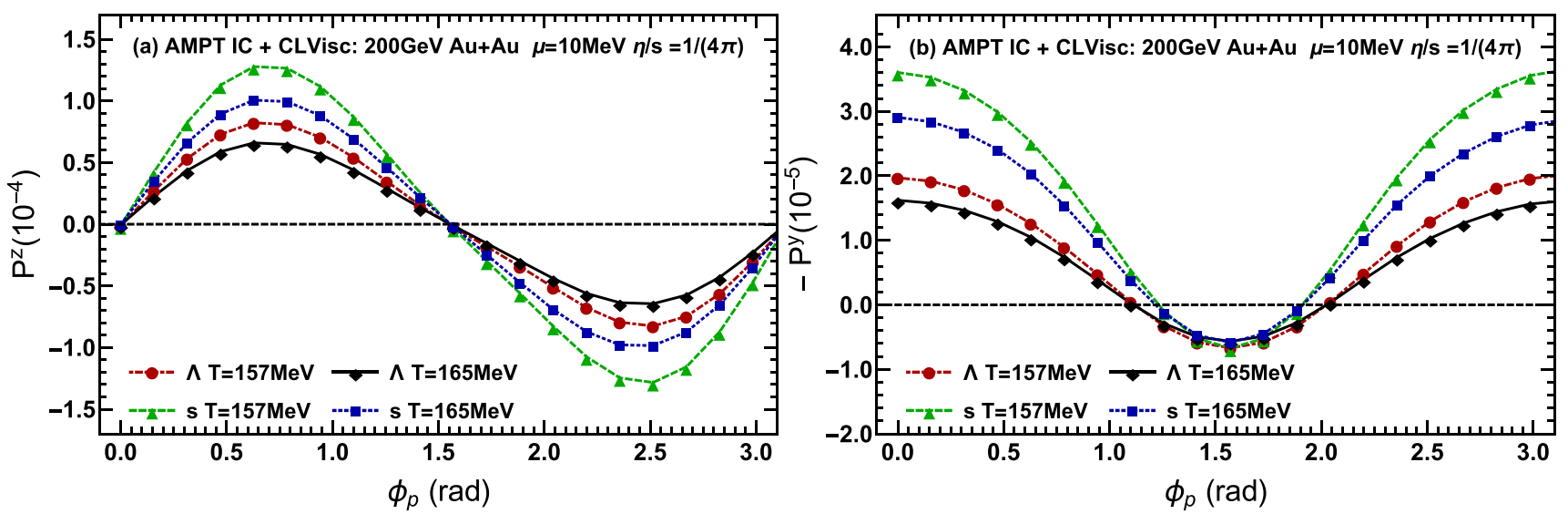

FIG. 5. The polarization $\mathcal{P}_{\text {chemcial }}^{z}$ and $\mathcal{P}_{\text {chemcial }}^{y}$ as a function of $\phi_{p}$ for the $\Lambda$ and $s$ equilibrium scenarios. We have chosen $\eta / s=1 /(4 \pi)$ and the freeze-out temperature is 165 or $157 \mathrm{MeV}$. See the color assignments for the results in the plots. 


\section{CONCLUSION}

In this work, we first review the spin polarization (pseudo-) vector $\mathcal{S}^{\mu}(\mathbf{p})$ derived from Wigner functions in Ref. [36]. We decompose $\mathcal{S}^{\mu}(\mathbf{p})$ into $\mathcal{S}_{\text {thermal }}^{\mu}, \mathcal{S}_{\text {shear }}^{\mu}, \mathcal{S}_{\text {accT }}^{\mu}, \mathcal{S}_{\text {chemical }}^{\mu}$, and $\mathcal{S}_{\mathrm{EB}}^{\mu}$, which are led by the thermal vorticity, shear viscous tensor, fluid acceleration minus the gradient of temperature, gradient of the chemical potential over the temperature, and electromagnetic fields, respectively. We then implement $(3+$ 1)-dimensional viscous hydrodynamic CLVisc with AMPT initial conditions to obtain the numerical results for local spin polarization with zero electromagnetic fields and a vanishing chemical potential and focus on $\mathcal{P}_{\text {thermal }}^{\mu}, \mathcal{P}_{\text {shear }}^{\mu}$, and $\mathcal{P}_{\text {accT }}^{\mu}$ contributed by $\mathcal{S}_{\text {thermal }}^{\mu}, \mathcal{S}_{\text {shear }}^{\mu}$, and $\mathcal{S}_{\text {accT }}^{\mu}$. Inspired by Ref. [89], we also consider $\Lambda$ and $s$ equilibrium scenarios. As opposed to Ref. [89], we have chosen the EoS "s95p-pce" as a different EoS and investigate the influence of $\eta / s$ upon spin polarization.

Our numerical results show that both $\mathcal{P}_{\text {shear }}^{\mu}$ and $\mathcal{P}_{\text {accT }}^{\mu}$ affect $\mathcal{P}_{\text {total }}^{\mu}$, in addition to $\mathcal{P}_{\text {thermal }}^{\mu}$, which has been well studied in the literature. Although $\mathcal{P}_{\text {shear }}^{\mu}$ can lead to the "same" sign contribution, qualitatively consistent with experimental data, to the local spin polarization, $\mathcal{P}_{\text {accT }}^{\mu}$ also plays a crucial role and could change the behavior of $\mathcal{P}_{\text {total }}^{\mu}$, especially with a lower freeze-out temperature. It turns out that $\mathcal{P}_{\text {total }}^{\mu}$ is rather sensitive to the EoS, $\eta / s$, and the freeze-out temperature $T$. With the EoS adopted in our study, we do not observe a similar azimuthal angle dependence of the local spin polarization as in the experimental data.

We conclude that although the shear-induced polarization alone may result in sizable effects on the local spin polarization qualitatively consistent with experimental observations, it may not always be the dominant effect over other contributions at local equilibrium.

Although we have also checked the total local spin polarization by using another EoS and gotten behavior similar to that in Ref. [89], the total local spin polarization in the $s$ equilibrium scenario could still be highly suppressed if one chooses $m_{\Lambda}$ instead of $m_{s}$ in the denominator on the right-hand side of Eq. (2).

We also note that Ref. [90] added the contribution from the shear viscous tensor to the local spin polarization and assumed isothermal equilibrium, in which the gradient of the temperature is assumed to be vanishing near the freeze-out hypersurface. It is also tempting to study the dependence of the EoS near isothermal equilibrium in the future.

Consequently, the so-called "sign" problem remains an open question. Moreover, from the theoretical perspective, the Wigner function at local equilibrium without the inclusion of off-equilibrium corrections pertinent to interaction is not a self-consistent solution for a kinetic equation. As indirectly supported by the influence of $\mathcal{P}_{\text {accT }}^{\mu}$, even under the nearequilibrium condition, the second-order gradient terms, such as the nonequilibrium corrections qualitatively studied in, e.g., Refs. [36] and [91] with chiral kinetic theory, may potentially give rise to sizable contributions in numerical simulations. To comprehensively investigate the spin polarization of fermions near local equilibrium and its direct connection to the sign problem, it will still be imperative to conduct theoretical and numerical studies of spin hydrodynamics and the QKT with collisions.

\section{ACKNOWLEDGMENTS}

We are grateful to Yi Yin, Longgang Pang, Shuai Y. F. Liu, Baochi Fu, Huichao Song, and Francesco Becattini for helpful discussions. S.P. was supported by the National Nature Science Foundation of China (NSFC) under Grants No. 12075235 and No. 12135011. D.-L.Y. was supported by the Ministry of Science and Technology, Taiwan, under Grant No. MOST 110-2112-M-001-070-MY3.
[1] Z.-T. Liang and X.-N. Wang, Phys. Rev. Lett. 94, 102301 (2005). [Erratum: 96, 039901 (2006)].

[2] Z.-T. Liang and X.-N. Wang, Phys. Lett. B 629, 20 (2005).

[3] F. Becattini and F. Piccinini, Ann. Phys. 323, 2452 (2008).

[4] F. Becattini, F. Piccinini, and J. Rizzo, Phys. Rev. C 77, 024906 (2008).

[5] F. Becattini, V. Chandra, L. Del Zanna, and E. Grossi, Ann. Phys. 338, 32 (2013).

[6] Y. Jiang, Z.-W. Lin, and J. Liao, Phys. Rev. C 94, 044910 (2016); [Erratum: 95, 049904 (2017)].

[7] W.-T. Deng and X.-G. Huang, Phys. Rev. C 93, 064907 (2016).

[8] H. Li, L.-G. Pang, Q. Wang, and X.-L. Xia, Phys. Rev. C 96, 054908 (2017).

[9] R.-H. Fang, L.-G. Pang, Q. Wang, and X.-N. Wang, Phys. Rev. C 94, 024904 (2016).

[10] D.-X. Wei, W.-T. Deng, and X.-G. Huang, Phys. Rev. C 99, 014905 (2019).

[11] L. P. Csernai, V. K. Magas, and D. J. Wang, Phys. Rev. C 87, 034906 (2013).
[12] F. Becattini, L. P. Csernai, and D. J. Wang, Phys. Rev. C 88, 034905 (2013); [Erratum: 93, 069901 (2016)].

[13] F. Becattini et al., Eur. Phys. J. C 75, 406 (2015); [Erratum: 78, 354 (2018)].

[14] L.-G. Pang, H. Petersen, Q. Wang, and X.-N. Wang, Phys. Rev. Lett. 117, 192301 (2016).

[15] H.-Z. Wu, L.-G. Pang, X.-G. Huang, and Q. Wang, Nucl. Phys. A 1005, 121831 (2021).

[16] B. Betz, M. Gyulassy, and G. Torrieri, Phys. Rev. C 76, 044901 (2007).

[17] Y. B. Ivanov and A. A. Soldatov, Phys. Rev. C 95, 054915 (2017).

[18] Y. B. Ivanov and A. A. Soldatov, Phys. Rev. C 97, 044915 (2018).

[19] Y. B. Ivanov, V. D. Toneev, and A. A. Soldatov, Phys. Rev. C 100, 014908 (2019).

[20] I. Karpenko and F. Becattini, Eur. Phys. J. C 77, 213 (2017).

[21] Y. Xie, D. Wang, and L. P. Csernai, Phys. Rev. C 95, 031901(R) (2017). 
[22] Y. Sun and C. M. Ko, Phys. Rev. C 96, 024906 (2017).

[23] S. Shi, K. Li, and J. Liao, Phys. Lett. B 788, 409 (2019).

[24] L. Adamczyk et al. (STAR Collaboration), Nature (London) 548, 62 (2017).

[25] J. Adam et al. (STAR Collaboration), Phys. Rev. Lett. 123, 132301 (2019).

[26] T. Niida (STAR Collaboration), Nucl. Phys. A 982, 511 (2019).

[27] F. Becattini and I. Karpenko, Phys. Rev. Lett. 120, 012302 (2018).

[28] B. Fu, K. Xu, X.-G. Huang, and H. Song, Phys. Rev. C 103, 024903 (2021)

[29] X.-L. Xia, H. Li, Z.-B. Tang, and Q. Wang, Phys. Rev. C 98, 024905 (2018)

[30] S. Y. F. Liu, Y. Sun, and C. M. Ko, Phys. Rev. Lett. 125, 062301 (2020).

[31] S. A. Voloshin, EPJ Web Conf. 171, 07002 (2018).

[32] H.-Z. Wu, L.-G. Pang, X.-G. Huang, and Q. Wang, Phys. Rev. Research 1, 033058 (2019).

[33] X.-L. Xia, H. Li, X.-G. Huang, and H. Z. Huang, Phys. Rev. C 100, 014913 (2019).

[34] F. Becattini, G. Cao, and E. Speranza, Eur. Phys. J. C 79, 741 (2019).

[35] R.-H. Fang, J.-Y. Pang, Q. Wang, and X.-N. Wang, Phys. Rev. D 95, 014032 (2017).

[36] Y. Hidaka, S. Pu, and D.-L. Yang, Phys. Rev. D 97, 016004 (2018).

[37] M. A. Stephanov and Y. Yin, Phys. Rev. Lett. 109, 162001 (2012).

[38] D. T. Son and N. Yamamoto, Phys. Rev. D 87, 085016 (2013).

[39] J.-W. Chen, S. Pu, Q. Wang, and X.-N. Wang, Phys. Rev. Lett. 110, 262301 (2013).

[40] C. Manuel and J. M. Torres-Rincon, Phys. Rev. D 89, 096002 (2014).

[41] C. Manuel and J. M. Torres-Rincon, Phys. Rev. D 90, 076007 (2014).

[42] J.-Y. Chen, D. T. Son, M. A. Stephanov, H.-U. Yee, and Y. Yin, Phys. Rev. Lett. 113, 182302 (2014).

[43] J.-Y. Chen, D. T. Son, and M. A. Stephanov, Phys. Rev. Lett. 115, 021601 (2015).

[44] Y. Hidaka, S. Pu, and D.-L. Yang, Phys. Rev. D 95, 091901(R) (2017).

[45] N. Mueller and R. Venugopalan, Phys. Rev. D 97, 051901(R) (2018).

[46] Y. Hidaka, S. Pu, and D.-L. Yang, Nucl. Phys. A 982, 547 (2019).

[47] A. Huang, S. Shi, Y. Jiang, J. Liao, and P. Zhuang, Phys. Rev. D 98, 036010 (2018).

[48] J.-H. Gao, Z.-T. Liang, Q. Wang, and X.-N. Wang, Phys. Rev. D 98, 036019 (2018).

[49] Y.-C. Liu, L.-L. Gao, K. Mameda, and X.-G. Huang, Phys. Rev. D 99, 085014 (2019).

[50] S. Lin and A. Shukla, J. High Energy Phys. 06 (2019) 060.

[51] S. Lin and L. Yang, Phys. Rev. D 101, 034006 (2020).

[52] N. Yamamoto and D.-L. Yang, Astrophys. J. 895, 56 (2020).

[53] J.-H. Gao and Z.-T. Liang, Phys. Rev. D 100, 056021 (2019).

[54] N. Weickgenannt, X.-L. Sheng, E. Speranza, Q. Wang, and D. H. Rischke, Phys. Rev. D 100, 056018 (2019).
[55] K. Hattori, Y. Hidaka, and D.-L. Yang, Phys. Rev. D 100, 096011 (2019).

[56] Z. Wang, X. Guo, S. Shi, and P. Zhuang, Phys. Rev. D 100, 014015 (2019).

[57] D.-L. Yang, K. Hattori, and Y. Hidaka, J. High Energy Phys. 07 (2020) 070.

[58] N. Weickgenannt, E. Speranza, X.-L. Sheng, Q. Wang, and D. H. Rischke, Phys. Rev. Lett. 127, 052301 (2021).

[59] N. Weickgenannt, X.-L. Sheng, E. Speranza, Q. Wang, and D. H. Rischke, Nucl. Phys. A 1005, 121963 (2021).

[60] S. Li and H.-U. Yee, Phys. Rev. D 100, 056022 (2019).

[61] Y.-C. Liu, K. Mameda, and X.-G. Huang, Chin. Phys. C 44, 094101 (2020).

[62] Z. Wang, X. Guo, and P. Zhuang, Eur. Phys. J. C 81, 799 (2021).

[63] N. Weickgenannt, E. Speranza, X.-L. Sheng, Q. Wang, and D. H. Rischke, Phys. Rev. D 104, 016022 (2021).

[64] X.-L. Sheng, N. Weickgenannt, E. Speranza, D. H. Rischke, and Q. Wang, Phys. Rev. D 104, 016029 (2021).

[65] Z. Wang and P. Zhuang, arXiv:2105.00915.

[66] J.-J. Zhang, R.-H. Fang, Q. Wang, and X.-N. Wang, Phys. Rev. C 100, 064904 (2019).

[67] W. Florkowski, B. Friman, A. Jaiswal, and E. Speranza, Phys. Rev. C 97, 041901(R) (2018).

[68] W. Florkowski, E. Speranza, and F. Becattini, Acta Phys. Polon. B 49, 1409 (2018).

[69] D.-L. Yang, Phys. Rev. D 98, 076019 (2018).

[70] F. Becattini, W. Florkowski, and E. Speranza, Phys. Lett. B 789, 419 (2019).

[71] W. Florkowski, R. Ryblewski, and A. Kumar, Prog. Part. Nucl. Phys. 108, 103709 (2019).

[72] K. Hattori, M. Hongo, X.-G. Huang, M. Matsuo, and H. Taya, Phys. Lett. B 795, 100 (2019).

[73] S. Bhadury, W. Florkowski, A. Jaiswal, A. Kumar, and R. Ryblewski, Phys. Lett. B 814, 136096 (2021).

[74] S. Shi, C. Gale, and S. Jeon, Phys. Rev. C 103, 044906 (2021).

[75] D. Montenegro, L. Tinti, and G. Torrieri, Phys. Rev. D 96, 076016 (2017).

[76] D. Montenegro, L. Tinti, and G. Torrieri, Phys. Rev. D 96, 056012 (2017); [Addendum: 96, 079901 (2017)].

[77] S. Li, M. A. Stephanov, and H.-U. Yee, Phys. Rev. Lett. 127, 082302 (2021).

[78] K. Fukushima and S. Pu, Relativistic decomposition of the orbital and the spin angular momentum in chiral physics and Feynman's angular momentum paradox, in Strongly Interacting Matter under Rotation, edited by F. Becattini, J. Liao, and M. Lisa, Lecture Notes in Physics, Vol 987 (Springer, Cham, 2021), pp. 381-396.

[79] K. Fukushima and S. Pu, Phys. Lett. B 817, 136346 (2021).

[80] A. D. Gallegos, U. Gürsoy, and A. Yarom, SciPost Phys. 11, 041 (2021).

[81] Q. Wang, Nucl. Phys. A 967, 225 (2017).

[82] F. Becattini and M. A. Lisa, Ann. Rev. Nucl. Part. Sci. 70, 395 (2020).

[83] F. Becattini, Polarization in relativistic fluids: A quantum field theoretical derivation, in Strongly Interacting Matter under Rotation, edited by F. Becattini, J. Liao, and M. Lisa, Lecture Notes in Physics, Vol. 987 (Springer, Cham, 2021), pp. 15-52.

[84] J.-H. Gao, G.-L. Ma, S. Pu, and Q. Wang, Nucl. Sci. Tech. 31, 90 (2020). 
[85] Y.-C. Liu and X.-G. Huang, Nucl. Sci. Tech. 31, 56 (2020).

[86] S. Y. F. Liu and Y. Yin, Phys. Rev. D 104, 054043 (2021).

[87] S. Y. F. Liu and Y. Yin, JHEP 07 (2021) 188.

[88] F. Becattini, M. Buzzegoli, and A. Palermo, Phys. Lett. B 820, 136519 (2021).

[89] B. Fu, S. Y. F. Liu, L. Pang, H. Song, and Y. Yin, Phys. Rev. Lett. 127, 142301 (2021).

[90] F. Becattini, M. Buzzegoli, A. Palermo, G. Inghirami, and I. Karpenko, arXiv:2103.14621.

[91] Y. Hidaka and D.-L. Yang, Phys. Rev. D 98, 016012 (2018).

[92] I. Karpenko and F. Becattini, Nucl. Phys. A 982, 519 (2019).

[93] L. Pang, Q. Wang, and X.-N. Wang, Phys. Rev. C 86, 024911 (2012).
[94] L.-G. Pang, H. Petersen, and X.-N. Wang, Phys. Rev. C 97, 064918 (2018).

[95] Z.-W. Lin, C. M. Ko, B.-A. Li, B. Zhang, and S. Pal, Phys. Rev. C 72, 064901 (2005).

[96] P. Huovinen and P. Petreczky, Nucl. Phys. A 837, 26 (2010).

[97] G. S. Denicol, C. Gale, S. Jeon, A. Monnai, B. Schenke, and C. Shen, Phys. Rev. C 98, 034916 (2018).

[98] J. Bloczynski, X.-G. Huang, X. Zhang, and J. Liao, Phys. Lett. B 718, 1529 (2013).

[99] W.-T. Deng and X.-G. Huang, Phys. Rev. C 85, 044907 (2012).

[100] V. Roy and S. Pu, Phys. Rev. C 92, 064902 (2015).

[101] S. Borsanyi et al., Phys. Lett. B 730, 99 (2014). 